\title{
Enhanced Long-Term Potentiation-Like Plasticity of the Trigeminal Blink Reflex Circuit in Blepharospasm
}

\author{
Angelo Quartarone, ${ }^{1}$ Antonino Sant'Angelo, ${ }^{1}$ Fortunato Battaglia, ${ }^{2}$ Sergio Bagnato, ${ }^{1,3}$ Vincenzo Rizzo, ${ }^{1}$ \\ Francesca Morgante, ${ }^{1}$ John C. Rothwell, ${ }^{4}$ Hartwig R. Siebner, ${ }^{5}$ and Paolo Girlanda ${ }^{1}$ \\ ${ }^{1}$ Departments of Neurosciences, Psychiatric and Anaesthesiological Sciences, University of Messina, 98125 Messina, Italy, ${ }^{2}$ Department of Physiology and \\ Pharmacology, City College University of New York Medical School, New York, New York 10012, ${ }^{3}$ Fondazione Istituto San Raffaele-G. Giglio, 90015 Cefalù \\ (Pa), Italy, ${ }^{4}$ Sobell Department of Neurophysiology, Institute of Neurology, Queen Square, London WC1N BG3, United Kingdom, and ${ }^{5}$ Department of \\ Neurology, Christian-Albrechts-University, D-24105 Kiel, Germany
}

Benign essential blepharospasm (BEB) is a focal cranial dystonia affecting eye closure. Here, we tested the hypothesis that BEB is associated with abnormal plasticity of the neuronal circuits mediating reflex blinks. In patients with BEB and healthy age-matched controls, we used the conditioning protocol introduced by Mao and Evinger (2001) to induce long-term potentiation (LTP)-like plasticity in trigeminal wide dynamic range neurons of the blink reflex circuit. High-frequency trains of electrical stimuli were repeatedly given over the right supraorbital nerve (SO) and timed to coincide with the R2 response elicited by a preceding SO stimulus. High-frequency stimulation (HFS) resulted in a long-lasting and input-specific potentiation of the R2 response in both groups, yet the facilitation of the $\mathrm{R} 2$ response was markedly increased in patients relative to controls. Botulinum toxin (BTX) injections in both orbicularis oculi muscles normalized the previously enhanced LTP-like plasticity of the R2 response. The increased responsiveness to HFS provides first-time evidence that LTP-like plasticity is increased in the trigeminal reflex circuit of patients affected by BEB. The results also show that the enhanced modifiability is not fixed in BEB, because BTX injections can transiently restore normal LTP-like plasticity. We propose that an abnormal corneal input induced by excessive blinking exacerbates increased LTP-like plasticity in BEB. BTX treatment removes the latter and restores plasticity toward normal values. Our results support the concept that maladaptive reorganization contributes to the pathophysiology of focal dystonias.

Key words: blepharospasm; blink reflex; focal dystonia; long-term potentiation; LTP; maladaptive plasticity; basal ganglia

\section{Introduction}

Benign essential blepharospasm (BEB) is a focal dystonia characterized by sustained, involuntary blinking and closure of the eyelids typically caused by spasms of the orbicularis oculi (OO) muscles (Fahn, 1988). Although the pathophysiology of BEB is still unknown, several recent studies of the blink reflex have given some important clues about the nature of this condition (Hallett, 2002; Rothwell and Huang, 2003). Affected individuals show a normal R1 and R2 response to single-pulse stimulation of the supraorbital nerve ( $\mathrm{SO}$ ), but paired-pulse stimulation reveals a shortening of the recovery cycle of the R2 response (Berardelli et al., 1985; Tolosa et al., 1988; Pauletti et al., 1993). Schicatano et al. (1997) introduced an animal model of BEB in which symptoms occurred after coupling subclinical depletion of dopamine (DA) in the basal ganglia with weakening of the OO muscles. They proposed that DA loss exaggerated the usual adaptive increase in the gain of the blink reflex that follows weakening of the OO. This results in excessive blinking and eventual spontaneous eyelid closure typical of BEB. It has been proposed that a similar pre-

\footnotetext{
Received April 29, 2005; revised Sept. 19, 2005; accepted 0ct. 16, 2005

Correspondence should be addressed to Dr. Angelo Quartarone, Department of Neuroscience, Clinica Neurologica

2, Policlinico Universitario, 98125 Messina, Italy. E-mail: angelo.quartarone@unime.it.

DOI:10.1523/JNEUROSCI.3948-05.2006

Copyright $\odot 2006$ Society for Neuroscience $\quad$ 0270-6474/06/260716-06\$15.00/0
}

existing abnormality in plasticity of the blink reflex circuits explains why some patients develop blepharospasm-like symptoms on the side contralateral to facial nerve palsy (Chuke et al., 1996). Supporting this view, paired-pulse inhibition of the blink reflex was shown to be attenuated in patients with residual facial weakness but not in patients who fully recovered facial strength (Syed et al., 1999).

In healthy individuals, Mao and Evinger (2001) demonstrated that repetitive bursts of high-frequency stimulation (HFS) to the SO can facilitate or attenuate the R2 response of the blink reflex, if HFS is appropriately paired with movement feedback from the eyelid (Mao and Evinger, 2001). A long-lasting facilitation of reflex blinks was observed if HFS coincided with the reflex blink (Mao and Evinger, 2001). Conversely, HFS provoked a longlasting suppression of subsequent reflex blinks when HFS preceded the reflex blink. No consistent modulation of the blink reflex occurred when HFS was given immediately after the reflex blink. The conditioning effect was always restricted to the trigeminal afferents conditioned by HFS. The characteristics of the after effects suggest that supraorbital HFS induced long-term potentiation (LTP) and long-term depression-like associative plasticity in the wide dynamic range neurons belonging to the blink reflex circuit (Mao and Evinger, 2001). These adaptive changes in the size of the blink reflex allow for dynamic adjustment of the gain of 
Table 1. Clinical details of patients with BEB

\begin{tabular}{|c|c|c|c|c|c|c|}
\hline \multirow[b]{2}{*}{ Patient } & \multirow[b]{2}{*}{ Age (years) } & \multirow[b]{2}{*}{ Gender } & \multirow{2}{*}{$\begin{array}{l}\text { Duration of disease } \\
\text { (years) }\end{array}$} & \multicolumn{2}{|c|}{$\begin{array}{l}\text { Marsden-Schachter scale } \\
\text { (1981) }\end{array}$} & \multirow{2}{*}{$\begin{array}{l}\text { Last treatment with } \\
\text { botulinum toxin }\end{array}$} \\
\hline & & & & Before BTX & After BTX & \\
\hline 1 & 67 & $M$ & 14 & 3 & 2 & 6 months \\
\hline 2 & 46 & $M$ & 5 & 4 & 1 & 4 months \\
\hline 3 & 70 & $\mathrm{~F}$ & 10 & 2 & 1 & 6 months \\
\hline 4 & 50 & M & 13 & 1 & 0 & 1 year \\
\hline 5 & 45 & $\mathrm{~F}$ & 10 & 2 & 1 & 7 months \\
\hline 6 & 79 & $\mathrm{~F}$ & 12 & 1 & 0 & 3 years \\
\hline 7 & 60 & M & 5 & 2 & 1 & 4 months \\
\hline 8 & 60 & M & 4 & 2 & 2 & 6 months \\
\hline 9 & 61 & $\mathrm{~F}$ & 4 & 3 & 2 & 4 months \\
\hline 10 & 67 & $\mathrm{~F}$ & 5 & 3 & 1 & 5 months \\
\hline 11 & 72 & $\mathrm{~F}$ & 11 & 1 & 0 & 6 months \\
\hline 12 & 72 & M & 12 & 1 & 0 & 6 months \\
\hline 13 & 59 & $\mathrm{~F}$ & 9 & 2 & 1 & 6 months \\
\hline 14 & 65 & $\mathrm{~F}$ & 10 & 2 & 1 & 7 months \\
\hline 15 & 81 & $\mathrm{~F}$ & 9 & 2 & 1 & 1 year \\
\hline 16 & 38 & M & 4 & 1 & 1 & No treatment \\
\hline
\end{tabular}

F, Female; M, male.

reflex blinks and thus help to maintain an appropriate relationship between sensory input (i.e., feedback from the eyelid movement) and motor output (i.e., blink amplitude) (Mao and Evinger, 2001).

The paradigm introduced by Mao and Evinger (2001) provides a unique opportunity to study plasticity-related phenomena in cranial dystonias. Here, we used the facilitatory HFS protocol to induce LTP-like plasticity in the blink reflex circuit in patients with BEB. Our prediction was that the blink reflex circuits would be more responsive to the facilitatory HFS protocol in patients with BEB compared with healthy controls.

\section{Materials and Methods}

Participants. Sixteen patients (nine females; $64.1 \pm 8.6$ years of age) with $\mathrm{BEB}$ and 11 normal subjects (five females; $59.2 \pm 12.3$ years of age) participated in the study. All participants gave their written informed consent, and experimental procedures were approved by the local Ethics Committee. The clinical details of the patients are summarized in Table 1. The severity of dystonia was assessed using the Marsden-Schachter Scale, severity factors, items for BEB before the experiment (Burke et al., 1985). All patients except one (patient $\left.n^{\circ} 16\right)$ (Table 1) had been treated previously with local injections of botulinum toxin type A (BTX) in the affected muscles, and none had any additional neurological disorder. The last BTX injection had been performed at least 4 months before the study.

Electrical stimulation of the SO nerve was performed using silver chloride disc surface electrodes. The cathode was placed over the supraorbital foramen and the anode $2 \mathrm{~cm}$ above. For electrical stimulation, we used a square wave pulse with a pulse width of $200 \mu \mathrm{s}$. The R2 threshold was determined as the minimum intensity required to evoke a reliable R2 response with an amplitude of $\geq 50 \mu \mathrm{V}$. Stimulus intensity was always set at two times the threshold to evoke a consistent $\mathrm{R} 2$ response $\left(2 \mathrm{~T}_{\mathrm{R} 2}\right)$. We used the HFS protocol introduced by Mao and Evinger (2001) to produce an LTP-like effect on the R2 component of the human blink reflex (supplemental figure, available at www.jneurosci.org as supplemental material). HFS was given in three blocks separated by 5 min. During each block, we gave four trains of HFS (nine stimuli per train) to the right SO nerve at a rate of $400 \mathrm{~Hz}$ and an intensity of $2 \mathrm{~T}_{\mathrm{R} 2}$. The intertrain interval was $10 \mathrm{~s}$. Each HFS train consisted of nine stimuli and was conditioned by a single $2 \mathrm{~T}_{\mathrm{R} 2}$ stimulus to the right SO nerve. According to the "DURING" condition described previously by Mao and Evinger (2001), HFS started at the onset of the R2 response elicited by the conditioning stimulus (supplemental figure, available at www.jneurosci.org as supplemental material).

To assess the conditioning effects of HFS, the area of the R2 compo- nent of the blink reflex was measured during four blocks of measurements immediately before HFS conditioning [i.e., baseline (B)], immediately after $\left(\mathrm{T}_{0}\right)$, and $30 \min \left(\mathrm{T}_{30}\right)$ and 60 $\min \left(\mathrm{T}_{60}\right)$ after HFS conditioning. For each block of measurements, we recorded the electromyographic (EMG) activity from both OO muscles during the blink reflex.

In eight patients (six females; mean age, $60 \pm$ 7 years) and eight healthy controls (five females; mean age, $56 \pm 9$ years), we additionally investigated how HFS conditioning of the right SO nerve changes the $\mathrm{R} 2$ response elicited by a 2 $\mathrm{T}_{\mathrm{R} 2}$ stimulus to the left $\mathrm{SO}$ nerve. Apart from contralateral (left) electrical stimulation of the SO nerve to induce a blink reflex, experimental procedures were kept identical to the main experiment. Both experiments were performed in a counterbalanced order on separate days at least $3 \mathrm{~d}$ apart. Finally, eight patients (five females; mean age, $62.3 \pm 7.2$ years) were reevaluated 4 weeks after they had been treated with BTX injections in both OO muscles (Dysport, 80-160 mouse units, four injection sites).

We were concerned that any difference between patients and controls may be caused by stronger reflex blinks in response to $\mathrm{SO}$ stimulation at $2 \mathrm{~T}_{\mathrm{R} 2}$. Therefore, we performed a separate experiment on five healthy individuals (four males; mean age, $55 \pm 9$ years) to compare the after effects of HFS conditioning using twofold (2 $\left.\mathrm{T}_{\mathrm{R} 2}\right)$ or threefold $\left(3 \mathrm{~T}_{\mathrm{R} 2}\right)$ threshold intensity for $\mathrm{SO}$ stimulation. The experiments were performed on separate days, at least 1 week apart, in a counterbalanced order. The area of the R2 component of the blink reflex was determined in blocks of measurements immediately before (i.e., baseline) and after HFS conditioning $\left(\mathrm{T}_{0}\right)$. Otherwise, the experimental procedures were identical to the main experiment.

Because blepharospasm caused increased background contraction in the OO muscle during HFS conditioning in patients but not in healthy controls, we were concerned that any difference in the response to HFS conditioning might be attributed to dystonic activity in the $\mathrm{OO}$ muscle at the time of conditioning. To address this point, we performed a control experiment in five healthy individuals (three males; mean age, $33.6 \pm 4.1$ years) to examine how continuous or intermittent activity in the $\mathrm{OO}$ muscle modifies the efficacy of HFS conditioning. Continuous muscular preactivation involved a slight tonic lid closure for $\sim 20 \mathrm{~s}$ with short opening periods to avoid sleepiness (open periods of $\sim 2-5$ s). Participants had to perform irregular blinks in response to a tone to produce intermittent muscular activity. Blinking activity was standardized by pacing the blinks at a rate between 0.5 and $2 \mathrm{~Hz}$. Continuous or intermittent preactivation of the $\mathrm{OO}$ muscles was maintained during the intervals between the high-frequency trains. The effects of HFS conditioning during continuous or intermittent preactivation were assessed in separate sessions in a counterbalanced order. The area of the R2 component of the blink reflex was determined in blocks of measurements immediately before (i.e., baseline) and after HFS conditioning $\left(\mathrm{T}_{0}\right)$.

Data acquisition and analysis. Reflex responses were recorded with pairs of silver chloride disc surface electrodes placed over both OO muscles. Twenty trials were collected and averaged for each block of measurements. Trials with movement artifacts were rejected. The EMG signal was amplified and bandpass filtered $(20 \mathrm{~Hz}$ to $3 \mathrm{KHz}$; D150 amplifier; Digitimer, Welwyn Garden City, Herts, UK) and stored at a sampling rate of $5 \mathrm{KHz}$ on a personal computer for off-line analysis (SigAvg Software; Cambridge Electronic Design, Cambridge, UK). The area of the R2 response was calculated for each block by integrating the rectified EMG activity of the OO muscles using NuCursor software (Sobell Research Department of Motor Neuroscience and Movement Disorders, Institute of Neurology, University College of London, London, UK). The onset and offset of the R2 response were estimated visually from averaged rectified EMG measures.

The primary measure was the EMG area of the $\mathrm{R} 2$ response in the right 
and left $\mathrm{OO}$ muscles. Using the area of the R2 response as a dependent variable, the effects of HFS conditioning were evaluated using ANOVA for repeated measurements. We computed an ANOVA with time (four levels, $\mathrm{B}, \mathrm{T}_{0}$, $\mathrm{T}_{30}$, and $\mathrm{T}_{60}$ ) and muscle (two levels, right vs left $\mathrm{OO}$ muscle) as within-subject factor and group (two levels, patients vs controls) as between-subjects factor.

In patients, the effects of botulinum toxin treatment on blink reflex potentiation was tested performing a separate ANOVA with time (four levels, $\mathrm{B}, \mathrm{T}_{0}, \mathrm{~T}_{30}$, and $\mathrm{T}_{60}$ ), treatment (two levels, before and after BTX), and muscle (two levels, right vs left $\mathrm{OO}$ muscle) as within-subject factors.

In healthy controls, the effects of stimulus intensity and muscular preactivation on HFS conditioning were assessed in separate ANOVAs using the relative change in R2 amplitude as the dependent variable. To test the effects of stimulus intensity, we performed an ANOVA, which treated the intensity of SO stimulation (two levels, $2 \mathrm{~T}_{\mathrm{R} 2}$ or $3 \mathrm{~T}_{\mathrm{R} 2}$ threshold intensity for SO stimulation) and muscle (two levels, right vs left OO muscle) as the within-subject factor. The effects of background activity in the $\mathrm{OO}$ muscle on the amount of R2 facilitation were assessed computing an ANOVA with type of preactivation (three levels, no preactivation, continuous preactivation, and intermittent preactivation) and muscle (two levels, right vs left $\mathrm{OO}$ muscle) as the within-subject factor.

The Greenhouse-Geisser method was used if necessary to correct for nonsphericity. Conditional on a significant $F$ value, post hoc paired-sample $t$ tests were used to explore the strength of main effects and the patterns of interaction between experimental factors. A post hoc Tukey's honest significant difference test was performed to explore the strength of main effects and the patterns of interaction between experimental factors. A $p$ value of $<0.05$ was considered significant. All data are given as mean \pm SEM.

\section{Results}

Subjects did not report any adverse side effects during the course of the study. Moreover, patients did not notice any significant effect of HFS in blepharospasm intensity and frequency.

The mean intensity used for stimulation of the right SO nerve did not differ between patients $(12.5 \pm 6.3 \mathrm{~mA})$ and controls $(11.5 \pm 5.3 \mathrm{~mA})$. There was also no between-groups difference in the size of the $\mathrm{R} 2$ response at baseline (right OO muscle: patients, $84.9 \pm 21 \mu \mathrm{V} / \mathrm{s}$; controls, $119 \pm 29.8 \mu \mathrm{V} / \mathrm{s} ; p=0.1$; left $\mathrm{OO}$ muscle: patients, $60.3 \pm 7.5 \mu \mathrm{V} / \mathrm{s}$; controls, $80 \pm 12 \mu \mathrm{V} / \mathrm{s} ; p=$ $0.08)$.

In both groups, HFS conditioning induced a bilateral increase in area of the $\mathrm{R} 2$ response when the blink reflex was evoked by electrical stimulation of the treated (right) SO nerve (Fig. 1). This was reflected by a main effect of time $\left(F_{(3,78)}=17.3 ; p<0.001\right)$ in the ANOVA. However, HFS conditioning induced a stronger increase in $\mathrm{R} 2$ area in patients relative to healthy controls. In healthy controls, there was a mean increase in R2 area of $\sim 20 \%$ (range, 5-50\%), whereas patients showed a mean R2 facilitation of $\sim 60 \%$ (range, $15-400 \%$ ) relative to baseline values (Fig. 1). This differential effect of HFS conditioning was confirmed by a time by group interaction $\left(F_{(3,78)}=5.37 ; p=0.002\right)$. ANOVA revealed no main effect of side and no interaction between side and the other factors, indicating that HFS produced a symmetrical potentiation of the $\mathrm{R} 2$ area in both $\mathrm{OO}$ muscles.

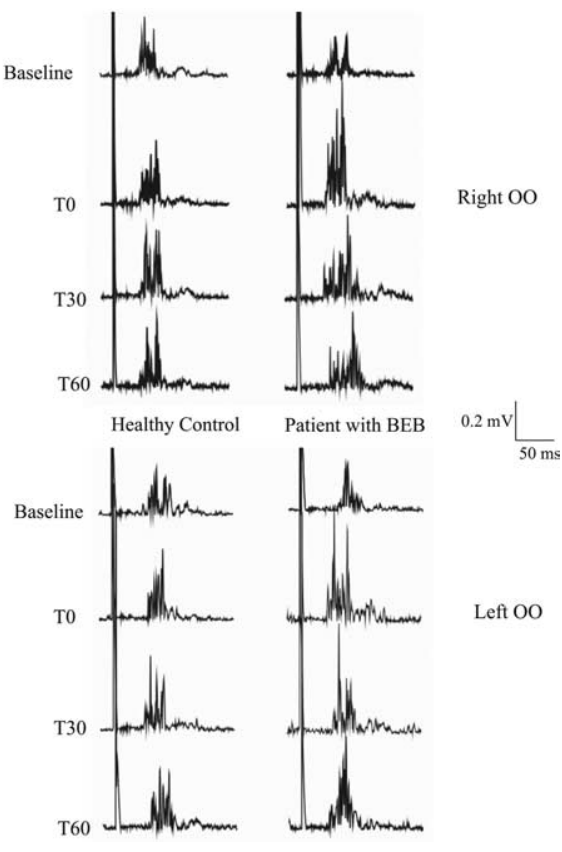

Time
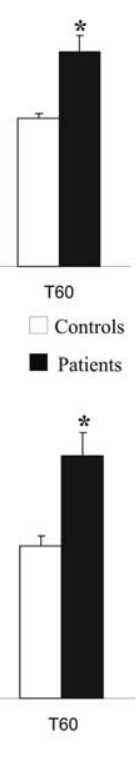

ft $\mathrm{OO}$

In separate follow-up ANOVAs, we explored the effects of HFS conditioning for each group. The ANOVA model included the factors time (four levels; baseline and $\mathrm{T}_{0}, \mathrm{~T}_{30}$, and $\mathrm{T}_{60}$ ) and side (two levels; right vs left $\mathrm{OO}$ muscle) as within-subject factors. For both groups, ANOVA demonstrated a main effect of time (patients: $F_{(3,45)}=16.42 ; p<0.00001$; controls: $F_{(3,33)}=6.01$; $p=0.002)$, but there was neither a main effect of side nor a time by side interaction. Pair-wise comparisons showed that in both groups, the $\mathrm{R} 2$ response was consistently increased at $\mathrm{T}_{0}, \mathrm{~T}_{30}$, and $\mathrm{T}_{60}$ relative to baseline $(p<0.01)$.

In eight patients and eight healthy controls, we additionally assessed the size of bilateral R2 responses elicited by electrical stimulation of the untreated (left) SO nerve before and after HFS conditioning of the right SO nerve. There was no main effect of time, side, and group and no interaction between these factors, indicating that HFS of the right SO nerve did not modify R2 responses after the stimulation of the untreated side (Fig. 2).

Eight patients received the same conditioning HFS 4 weeks after BTX treatment. BTX injections produced no significant change in R2 area (right OO muscle: before BTX injections, $99 \pm$ $24 \mu \mathrm{V} / \mathrm{s}$; after BTX injections, $75 \pm 7 \mu \mathrm{V} / \mathrm{s} ; p=0.3$; left $\mathrm{OO}$ muscle: before BTX injections, $58 \pm 10 \mu \mathrm{V} / \mathrm{s}$; after BTX injections, $55 \pm 21 \mu \mathrm{V} / \mathrm{s} ; p=0.78$ ), but BTX treatment resulted in a marked attenuation of HFS-induced facilitation of the R2 area that was paralleled by significant clinical improvement. Indeed, BEB patients showed significant improvement after BTX injection (Marsden and Schachter Scale score before BTX, median 2; after BTX, median 1; Wilcoxon test, $p<0.002$ ) (Fig. 3, Table 1).

This was confirmed by the ANOVA, which showed a significant time by treatment interaction $\left(F_{(3,21)}=4.02 ; p=0.02\right)$. We computed an additional ANOVA to compare R2 measurements 


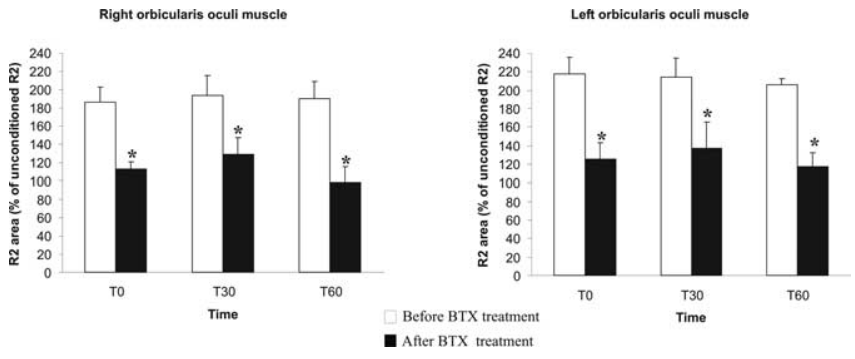

Figure 2. Blink reflexes by stimulating left untreated side. No conditioning effect of right $\mathrm{SO}$ nerve HFS is present. In healthy subjects (open columns) and patients with BEB (filled columns), HFS conditioning of the right $\mathrm{SO}$ nerve produced no lasting changes in the size of the R2 response when reflex blinks were elicited by stimulation of the untreated $\mathrm{SO}$ nerve. The bar chart gives the relative change in mean R2 area of both eyelids at T0, T30, and T60. Mean R2 area is expressed as a percentage of the R2 response at baseline. Each error bar represents SEM.

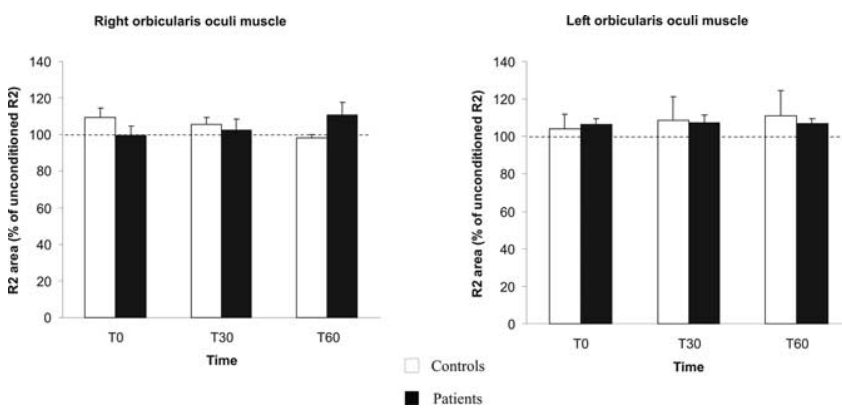

Figure 3. Effect of BTX treatment on HFS-induced increase in blink reflex excitability. In eight patients with $B E B$, the conditioning effects of HFS to the right $S 0$ nerve were assessed before (open columns) and 1 month after BTX treatment (filled columns). Reflex blinks were elicited by a $2 \mathrm{~T}$ stimulus given to the right $\mathrm{SO}$ nerve. The bar chart plots mean R2 areas of both eyelids at T0, T30, and T60. Mean R2 area is expressed as a percentage of the unconditioned R2 response. Each error bar represents SEM. Blink reflex potentiation was markedly reduced after BTX treatment. ${ }^{*} p<0.05$.

after BTX injections in patients with R2 measurements of eight age-matched healthy controls. This ANOVA showed no time by group interaction $\left(F_{(3,42)}=0.43 ; p=0.7\right)$, indicating a normal amount of R2 facilitation in BTX-treated patients.

In five healthy controls, electrical stimulation of the right $\mathrm{SO}$ nerve was used to elicit an R2 response before and immediately after HFS conditioning over the right SO nerve. HFS conditioning was given at an intensity of $2 \mathrm{~T}_{\mathrm{R} 2}$ or $3 \mathrm{~T}_{\mathrm{R} 2}$, whereas an intensity of $2 \mathrm{~T}_{\mathrm{R} 2}$ was always used for preconditioning and postconditioning measurements of the $\mathrm{R} 2$ response. Response size was measured bilaterally. An SO stimulus at $3 \mathrm{~T}_{\mathrm{R} 2}$ resulted in a stronger $\mathrm{R} 2$ response compared with an $\mathrm{SO}$ stimulus at $2 \mathrm{~T}_{\mathrm{R} 2}$ (mean R2 amplitude at $2 \mathrm{~T}_{\mathrm{R} 2}, 0.15 \pm 0.05$; mean $\mathrm{R} 2$ amplitude at $3 \mathrm{~T}_{\mathrm{R} 2}$, $\left.0.28 \pm 0.01 ; \mathrm{T}_{(1,8)}=-3.3 ; p=0.01\right)$. An increase in stimulus intensity to $3 \mathrm{~T}_{\mathrm{R} 2}$ did not enhance the conditioning effect of HFS in healthy controls (Fig. 4). Accordingly, ANOVA showed no main effect of stimulus intensity and no interaction between stimulus intensity and muscle.

In five healthy controls, we determined the effect of continuous or intermittent preactivation on the amount of $\mathrm{R} 2$ facilitation induced by HFS. Preactivation of the OO muscles during HFS conditioning did not increase the conditioning effect of HFS (Fig. 5). A two-factorial ANOVA showed no effect of preactivation and no interaction between preactivation and muscle.

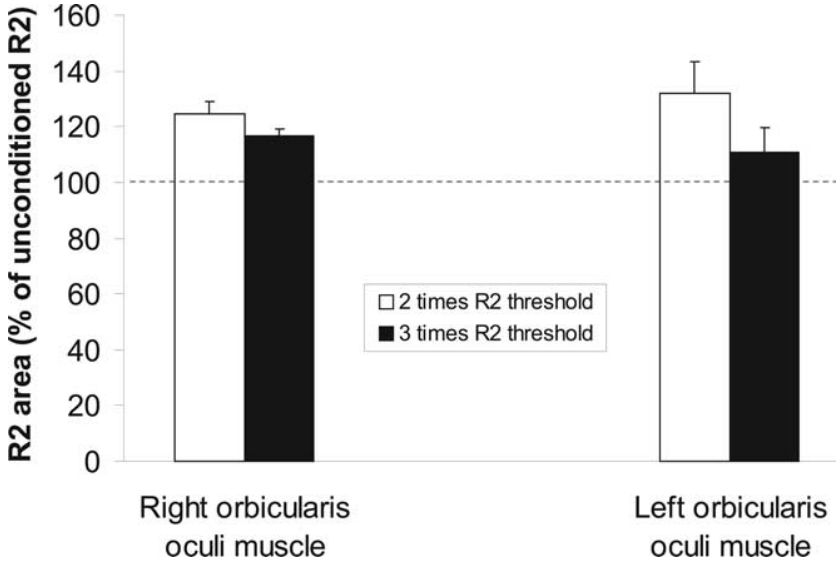

Figure 4. Potentiation of reflex blinks induced by HFS conditioning at two stimulus intensities. The bar chart gives the relative change in mean R2 areas of both eyelids immediately after administration of the HFS protocol. The intensity for HFS was set at 200\% (open columns) or $300 \%$ (filled columns) of the R2 threshold. Mean R2 area is expressed as a percentage of the unconditioned R2 response. Each error bar represents SEM.

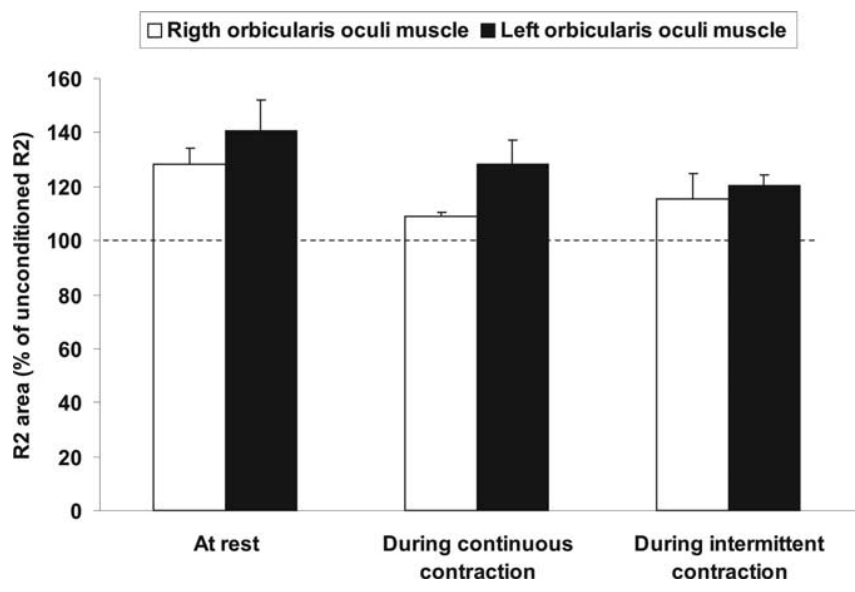

Figure 5. Potentiation of reflex blinks induced by HFS conditioning during preactivation of the 00 muscle. The bar chart plots the relative change in mean R2 areas before (open columns) and immediately after (filled columns) HFS conditioning during continuous or intermittent contraction of the 00 muscle in five healthy subjects. Mean R2 area is expressed as a percentage of the unconditioned R2 response. Each error bar represents SEM.

\section{Discussion}

Confirming the study by Mao and Evinger (2001), HFS conditioning of the SO nerve produced long-lasting facilitation of the trigeminal blink reflex if HFS was appropriately timed to coincide with an electrically induced $\mathrm{R} 2$ response. The facilitatory effect on reflex blinks evolved rapidly and was stable for a minimum of $1 \mathrm{~h}$. The after effect was topographically specific, because only eye blinks evoked by supraorbital stimulation on the treated side were facilitated. These features are compatible with LTP-like plasticity as suggested by Mao and Evinger (2001).

In patients with $\mathrm{BEB}$ and healthy controls, HFS conditioning of the right SO produced similar conditioning effects in terms of the direction, time course, and input specificity. The critical new finding was that untreated patients with BEB showed a stronger potentiation of the blink reflex than healthy controls. This is, to our knowledge, the first demonstration that BEB is associated with an increased modifiability of trigeminally evoked reflex blinks.

The increased response to HFS conditioning cannot be as- 
cribed to stronger reflex blinks in BEB patients relative to healthy subjects. First, the area of the R2 response did not differ between patients and healthy controls. This implies that there was no difference in movement feedback between groups at the time of HFS conditioning. Second, in five healthy volunteers, HFS conditioning at an intensity of $3 \mathrm{~T}_{\mathrm{R} 2}$ did not increase the amount of blink potentiation compared with HFS at an intensity of $2 \mathrm{~T}_{\mathrm{R} 2}$.

Another possible explanation relates to the fact that patients with cranial dystonia have an increased background contraction associated with involuntary movements in facial muscles. Thus, it is conceivable that the greater plasticity of the blink reflex after HFS in BEB was caused by the increased background contraction of the $\mathrm{OO}$ muscle. However, this is unlikely because in an additional control experiment performed in healthy subjects, we showed that preactivation of the OO muscles did not affect the degree of R2 facilitation after HFS conditioning.

The enhanced modifiability of the $\mathrm{R} 2$ response in patients with $\mathrm{BEB}$ is consistent with an increasing body of research suggesting that focal task-specific dystonias of the hand result from aberrant sensorimotor plasticity (Berardelli et al., 1998; Hallett, 1998; Quartarone et al., 2003, 2005). In monkeys, rapid, repetitive, stereotypical movements in a learning context can actively degrade the cortical representations of sensory information that guide fine hand movements (Byl et al., 1996). In patients with writer's cramp, a focal hand dystonia affecting handwriting, several recent studies have shown an enhanced responsiveness of the motor cortex to the conditioning effects of transcranial cortex stimulation (Hallett, 2002; Rothwell and Huang, 2003; Siebner et al., 2003). The recent study by Quartarone et al. (2003) on patients with writer's cramp has particular parallels with the present work. They used a sensorimotor conditioning protocol in which low-frequency stimulation of the right median nerve was followed by suprathreshold transcranial magnetic stimulation of the contralateral hand area at an interstimulus interval of $25 \mathrm{~ms}$ [paired associative stimulation (PAS)]. In healthy volunteers, this PAS protocol induces LTP-like increases in the amplitude of the motor-evoked potential elicited by transcranial magnetic stimulation (Stefan et al., 2002; Quartarone et al., 2003; Wolters et al., 2003). In an analogy to the present study, Quartarone et al. (2003) reported that facilitation was stronger in patients with writer's cramp (Quartarone et al., 2003). We speculate that enhanced modifiability of sensorimotor circuits may be characteristic of many types focal dystonia, perhaps at many different levels of the CNS.

It remains to be clarified which mechanism drives the enhanced response to HFS conditioning in patients with BEB. As initially proposed by Schicatano et al. (1997) in their animal model of BEB, endogenous factors such as a latent dysfunction of the basal ganglia may create a permissive environment for maladaptive plasticity to occur (Hallett, 1998). Small adjustments of the blink to changes in sensory input that occur in healthy subjects could become magnified in this environment and trigger symptoms of increased blinking. Indeed, if excess blinking induces additional irritation of the cornea, this could lead to a vicious circle of additional increases in blink gain and eventual appearance of spontaneous eyelid closure.

BTX treatment normalized the modifiability of the blink reflex in BEB, such that HFS conditioning potentiated the blink reflex to the same extent as in healthy subjects. At first sight, this normalization of blink "plasticity" seems at odds with previous work showing that BTX fails to reduce the heightened excitability of the blink in BEB as reflected in reduced prepulse inhibition and the enhanced recovery cycle of the R2 component (Girlanda et al., 1996). However, this may be related to the fact that two types of adaptation occur in the blink reflex circuit. Schicatano et al. (2002) examined the adaptive changes of reflex blinks during and shortly after a $2 \mathrm{~h}$ period of unilateral restraint of the upper lid in healthy subjects and showed that there were two distinct types of motor adaptation driven by two distinct error signals. In one, the difference between the actual and the planned blink magnitude created a proprioceptive error signal that drove an adaptive increase in excitability of ipsilateral motoneurons controlling the restrained eyelid. This led to increased blinks in the ipsilateral eye after stimulation of ipsilateral or contralateral SO nerve. In the second, a reduced blink amplitude caused corneal irritation. The corneal error signal produced an increased excitability of trigeminal reflex blink circuits and bilaterally enhanced blinks after stimulation of the ipsilateral but not contralateral SO nerve.

Mao and Evinger (2001) proposed that the modification of the blink reflex by HFS was caused by long-term changes in the responsiveness to inputs from the SO nerve of wide dynamic range (WDR) neurons in the trigeminal complex. We suggest that there is an endogenous increase in the plasticity of this circuit in BEB. However, increased blinking in BEB may itself reinforce this tendency by irritating the cornea and causing increased corneal input to the WDR neurons, making them more susceptible to conditioning with HFS. Treatment with BTX reduces blinking, reduces the corneal error signal and hence restores plasticity of the trigeminal complex toward normal values. However, at the same time, muscle weakness leads to an increase in the proprioceptive error signal, because each blink causes less movement than expected from a given motor output. This increased error feeds into the ipsilateral motoneurons and maintains the amplitude of the blink reflex after BTX despite the reduced plasticity of the circuit as demonstrated by HFS.

\section{References}

Berardelli A, Rothwell JC, Day BL, Marsden CD (1985) Pathophysiology of blepharospasm and oromandibular dystonia. Brain 108:593-608.

Berardelli A, Rothwell JC, Hallett M, Thompson PD, Manfredi M, Marsden CD (1998) The pathophysiology of primary dystonia. Brain 121:1195-1212.

Burke RE, Fahn S, Marsden CD, Bressman SB, Moskowitz C, Friedman J (1985) Validity and reliability of a rating scale for the primary torsion dystonias. Neurology 35:73-77.

Byl NN, Merzenich MM, Jenkins WM (1996) A primate genesis model of focal dystonia and repetitive strain injury: I. Learning-induced dedifferentiation of the representation of the hand in the primary somatosensory cortex in adult monkeys. Neurology 47:508-520.

Chuke JC, Baker RS, Porter JD (1996) Bell's Palsy-associated blepharospasm relieved by aiding eyelid closure. Ann Neurol 39:263-268.

Fahn S (1988) Blepharospasm: a form of focal dystonia. Adv Neurol 49:125-133.

Girlanda P, Quartarone A, Sinicropi S, Nicolosi C, Messina C (1996) Unilateral injection of botulinum toxin in blepharospasm: single fiber electromyography and blink reflex study. Mov Disord 11:27-31.

Hallett M (1998) Physiology of dystonia. Adv Neurol 78:11-18.

Hallett M (2002) Blepharospasm: recent advances. Neurology 59:1306-1312.

Mao JB, Evinger C (2001) Long-term potentiation of the human blink reflex. J Neurosci 21:RC151(1-4).

Pauletti G, Berardelli A, Cruccu G, Agostino R, Manfredi M (1993) Blink reflex and the masseter inhibitory reflex in patients with dystonia. Mov Disord 8:495-500.

Quartarone A, Bagnato S, Rizzo V, Siebner HR, Dattola V, Scalfari A, Morgante F, Battaglia F, Romano M, Girlanda P (2003) Abnormal associative plasticity of the human motor cortex in writer's cramp. Brain 126:2586-2596.

Quartarone A, Rizzo V, Bagnato S, Morgante F, Sant'Angelo A, Romano M, Crupi D, Girlanda P, Rothwell JC, Siebner HR (2005) Homeostatic-like plasticity of the primary motor hand area is impaired in focal hand dystonia. Brain 128:1943-1950. 
Rothwell JC, Huang YZ (2003) Systems-level studies of movement disorders in dystonia and Parkinson's disease. Curr Opin Neurobiol 13:691-695.

Schicatano EJ, Basso MA, Evinger C (1997) Animal model explains the origins of the cranial dystonia benign essential blepharospasm. J Neurophysiol 77:2842-2846.

Schicatano EJ, Mantzouranis J, Peshori KR, Partin J, Evinger C (2002) Lid restraint evokes two types of motor adaptation. J Neurosci 22:569-576.

Siebner HR, Filipovic SR, Rowe JB, Cordivari C, Gerschlager W, Rothwell JC, Frackowiak RS, Bhatia KP (2003) Patients with focal arm dystonia have increased sensitivity to slow-frequency repetitive TMS of the dorsal premotor cortex. Brain 126:2710-2725.
Stefan K, Kunesch E, Benecke R, Cohen LG, Classen J (2002) Mechanisms of enhancement of human motor cortex excitability induced by interventional paired associative stimulation. J Physiol (Lond) 543:699-708.

Syed NA, Delgado A, Sandbrink F, Schulman AE, Hallett M, Floeter MK (1999) Blink reflex recovery in facial weakness: an electrophysiologic study of adaptive changes. Neurology 52:834-838.

Tolosa E, Montserrat L, Bayes A (1988) Blink reflex studies in patients with focal dystonias. Adv Neurol 50:517-524.

Wolters A, Sandbrink F, Schlottmann A, Kunesch E, Stefan K, Cohen LG, Benecke R, Classen J (2003) A temporally asymmetric Hebbian rule governing plasticity in the human motor cortex. J Neurophysiol 89:23392345. 